Case Reports

\title{
Retroperitoneal Hydatid Cyst Masquerading as a Retroperitoneal Cystic Tumor
}

\author{
K. Hemanth Kumar, Anish Choudhary, Amaresh Aruni and N.V.K.N. Karthik
}

General Surgery, PGIMER, India

Article history

Received: 27-05-2019

Revised: 04-07-2019

Accepted: 26-08-2019

Corresponding Author: K. Hemanth Kumar General Surgery, PGIMER, India

Email: hemanthkr21@gmail.com
Abstract: Hydatid disease is an endemic disease, with canines the primary host and humans the intermediate host. Liver, spleen and lungs are most common involved organs, but it can involve any organ. Retroperitoneal hydatid cyst are categorised into two types, primary and secondary. Primary retroperitoneal hydatid cyst is rare disease, with most of the cases diagnosed intra-operatively, hence pre-operative high index of suspicion for any retroperitoneal cystic lesion to prevent inadvertent complication intra-operatively is of utmost important. 25year male patient presented with right flank pain for 3 months, high grade fever for 10 days. On cross sectional imaging a retroperitoneal cystic lesion of size $7.1 \times 7.6 \mathrm{~cm}$ was noted. Leucocytosis was present and rest of the blood parameters were with in normal limits. Patient was planned for surgery with a diagnosis of the retroperitoneal cystic tumour with possible tumour degeneration. On exploration $8 \times 8 \mathrm{~cm}$ pelvic retroperitoneal hydatid with daughter cysts and purulent material noted. Diagnosis of infected retroperitoneal hydatid cyst was made and cyst de-roofing and drainage was done. Final diagnosis of hydatid cyst was confirmed by histopathology report. The retroperitoneal hydatid cyst is a rare entity even in endemic areas. First reported in 1958 by Lockhart and Sapinza, an isolated retroperitoneal hydatid cyst could be caused by haematogenous dissemination of protoscoleces after bypassing the liver (venovenous shunts within the liver and in the space of Retzius) and the lungs or by the gastrointestinal tract into the lymphatic system. Dew and Waddle had favoured airborne transmission and direct implantation of the embryo in the bronchial mucosa as another possible mode of entry. This raises the possibility of an embryo of the parasite entering a venule after penetrating the bronchial mucosa and reaching the left side of the heart to involve other sites and thus bypassing the lung. Spontaneous, traumatic, or surgical rupture of a hepatic cyst may also give rise to cysts in retroperitoneum. The definitive diagnosis of a retroperitoneal hydatid cyst requires a combined assessment of clinical, radiological and serological analysis. Definitive treatment is total cystopericystectomy. Though retoperitoneal hydatid cyst are rare, diagnosis should be considered if any retroperitoneal cyst lesion is noted to prevent the complications of the cyst rupture. The gold standard treatment is total cystopericystectomy. If complete resection is not possible, cyst deroofing, drainage and adjuvant anti-helminthic should be considered.

Keywords: Retroperitoneum, Hydatid Cyst, Daughter Cysts 


\section{Introduction}

Hydatid disease is an endemic disease, with canines the primary host and humans the intermediate host. The life cycle of Echinococcus granulosus may also involve sheep, cattle, goats and humans. This infection is transmitted orally via eggs shed in the faeces of infected animals. Liver, spleen and lungs are most common involved organs but it can involve any organ. Retroperitoneal hydatid cyst are categorised in to two types, primary and secondary (Desai et al., 1999: Sherwani et al., 2015)

Primary retroperitoneal hydatid cyst is extremely rare and only occasional case reports have appeared since Lockhart and Sapinza first reported this entity in 1958. (Sherwani et al., 2015) $85 \%$ to $90 \%$ of patients with Echinococcus granulosus infection have single-organ involvement and more than $70 \%$ of patients have only one cyst. (Ozturk et al., 2014) The cysts may be uni or multiloculated and thin or thick walled. The clinical features are non-specific, most common symptom is abdomen pain, related to the size of the cyst \& it's pressure effects on surrounding structures, other cause of pain is related to the parietal peritoneum irritation due to the inflammatory reaction around the cyst wall. (Erdem et al., 2009) HD in extrahepatic locations especially in the retroperitoneum usually remains asymptomatic unless the cyst grows and produces symptoms due to pressure, rupture in to the pleural or peritoneal cavity, secondary infection or an allergic reaction (Avc1 et al., 2013).

\section{Case Presentation}

A 25 year old male patient had complaints of right flank pain for past 3 months associated with occasional episodes of nausea and vomiting. For the past 10 days preceding admission he had been having episodes of high grade fever on and off which brought him to us. There was no other GI symptoms. He gave history of appendectomy for acute appendicitis 1 year previous to the onset of symptoms. On examination a firm nontender mass of size $10 \times 8 \mathrm{~cm}$ was felt in the right iliac fossa. No other significant clinical finding was noted except scar of previous appendectomy at right iliac fossa.

Complete blood count showed elevated leukocyte count, no eosinophilia, liver and kidney function tests were normal. Plain X- ray abdomen did not show any specific diagnostic finding. USG abdomen revealed a well-defined globular lesion in RIF of size $6.7 \times 7.2 \mathrm{~cm}$ with no anechoic content, no significant vascularity or calcifications within. CECT abdomen showed a well defined retroperitoneal cystic lesion of size $7.1 \times 7.6 \mathrm{~cm}$ with involvement of the right iliopsoas (Fig. 1). On colonoscopy, a smooth bulge noted in ileum but mucosa was normal, rest of large bowel and terminal ileum was normal.

A provisional diagnosis of retroperitoneal cystic tumour was made and pseudomyxoma peritonei of the missed appendix tumor was kept as next differential diagnosis. The patient underwent an exploratory laparotomy through lower midline incision. A large $8 \times 8$ $\mathrm{cm}$ cystic lesion was identified in right lower retroperitoneum, displacing ureter and colon medially (Fig. 2). On exposing the posterior peritoneum cyst rupture was noted, however there was no spillage in to the surrounding structures. Around $250 \mathrm{~mL}$ of pus and daughter cysts were aspirated (Fig. 3 and 4). A diagnosis of infected hydatid cyst was made which was probably the culprit for the high grade fever with chills. The cyst cavity extended superiorly to right lumbar region, inferiorly to the pelvis. Cyst wall was de-roofed and irrigated with $10 \%$ betadine before closing abdomen and drains were placed in cyst cavity.

The patient was discharged on post-op day-5. Post-op albendazole was advised. Pathological examination of the specimen was reported as hydatid cyst (Fig. 5). Hydatid serology done retrospectively revealed a titre of $1: 1600$. Patient was followed up at 3,6 and 12 months with imaging, no evidence of recurrence noted.

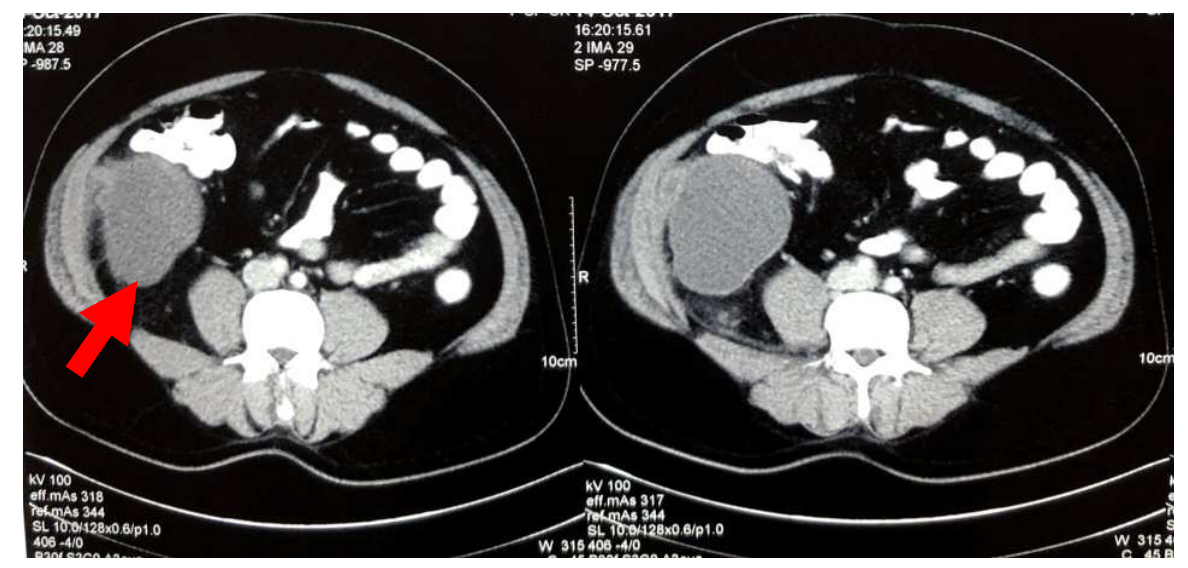

Fig. 1: CECT abdomen showing retroperitoneal cystic lesion (arrow) 


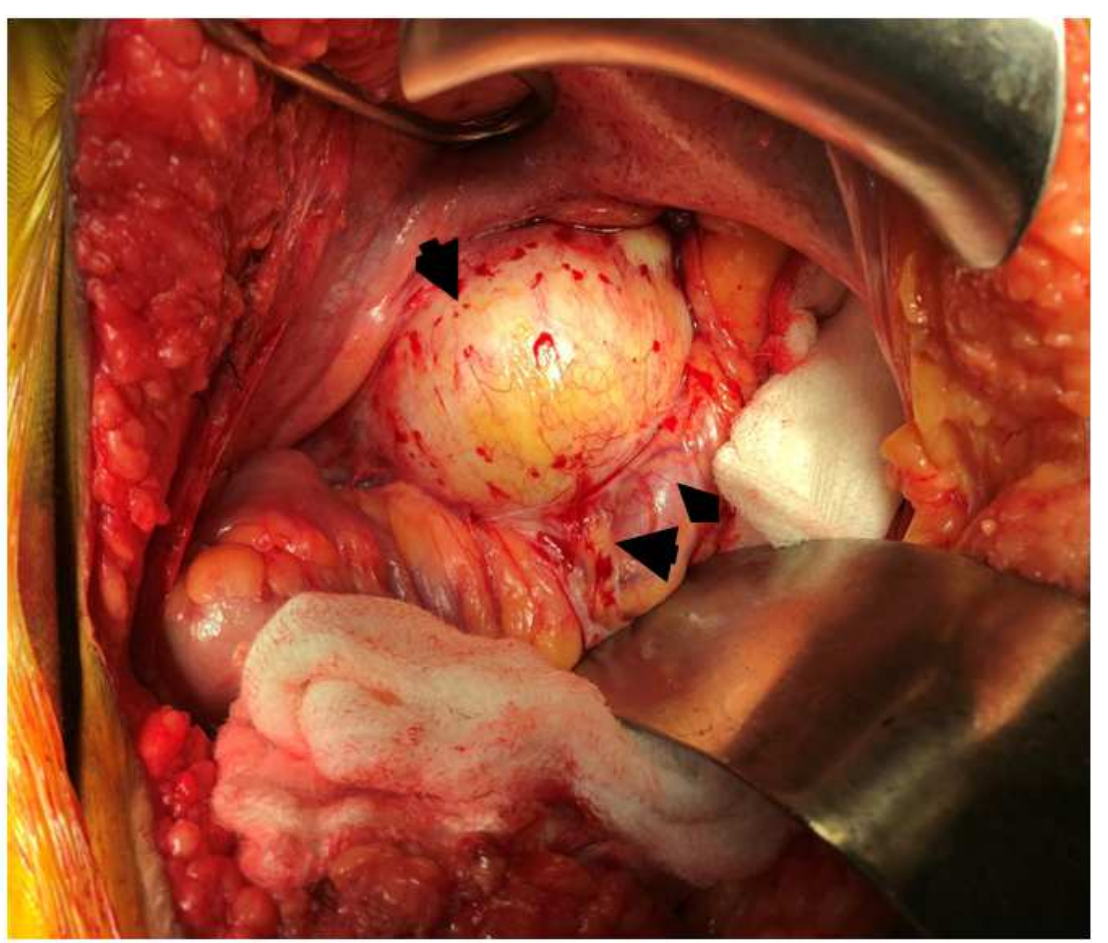

Fig. 2: $8 \times 8 \mathrm{~cm}$ cystic lesion in the pelvis retroperitoneum pushing the colon anteriorly. (cyst $=$ arrow head), (colon= double arrow head)

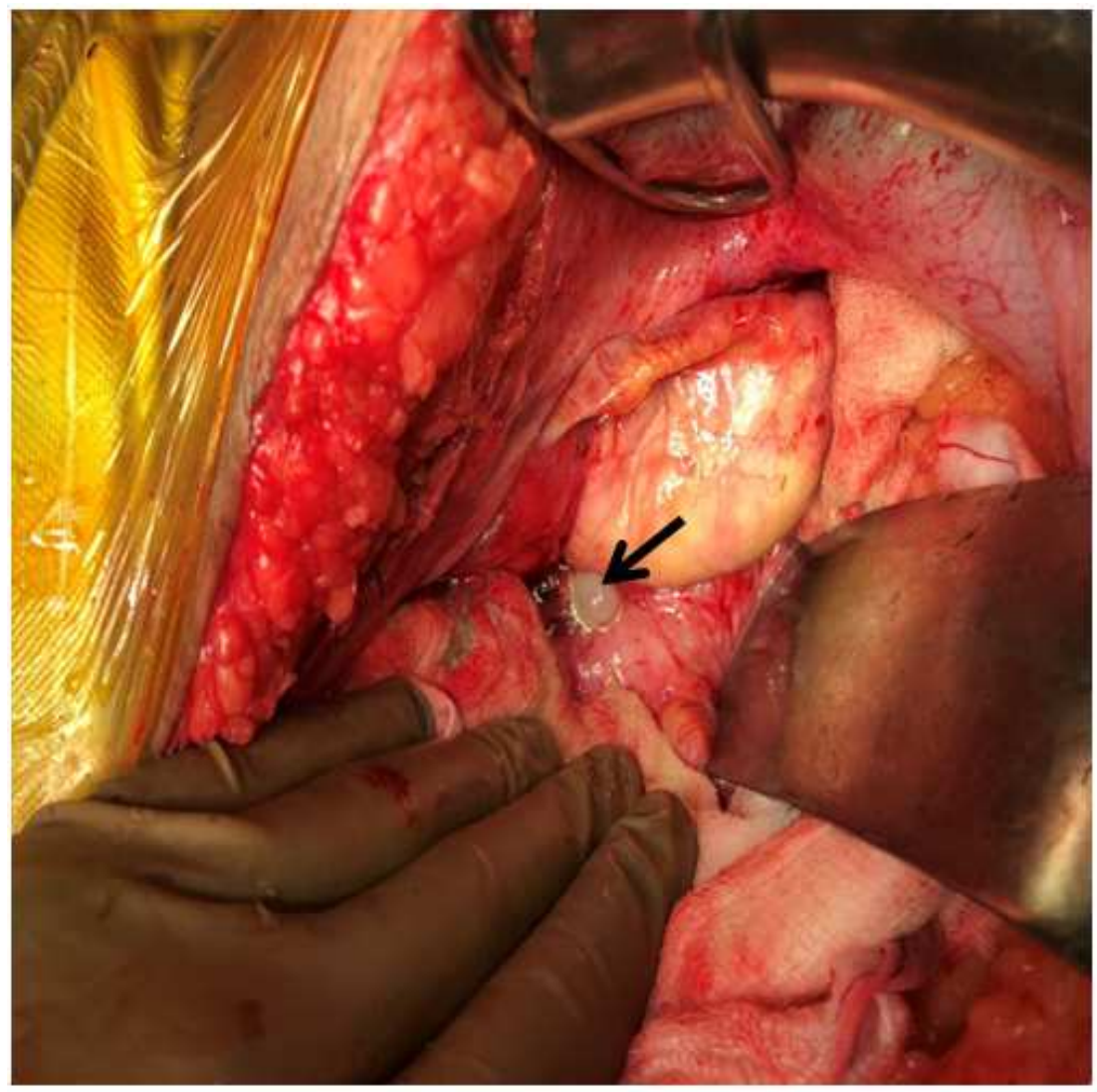

Fig. 3: Daughter cyst noted following iatrogenic rupture (Black arrow) 


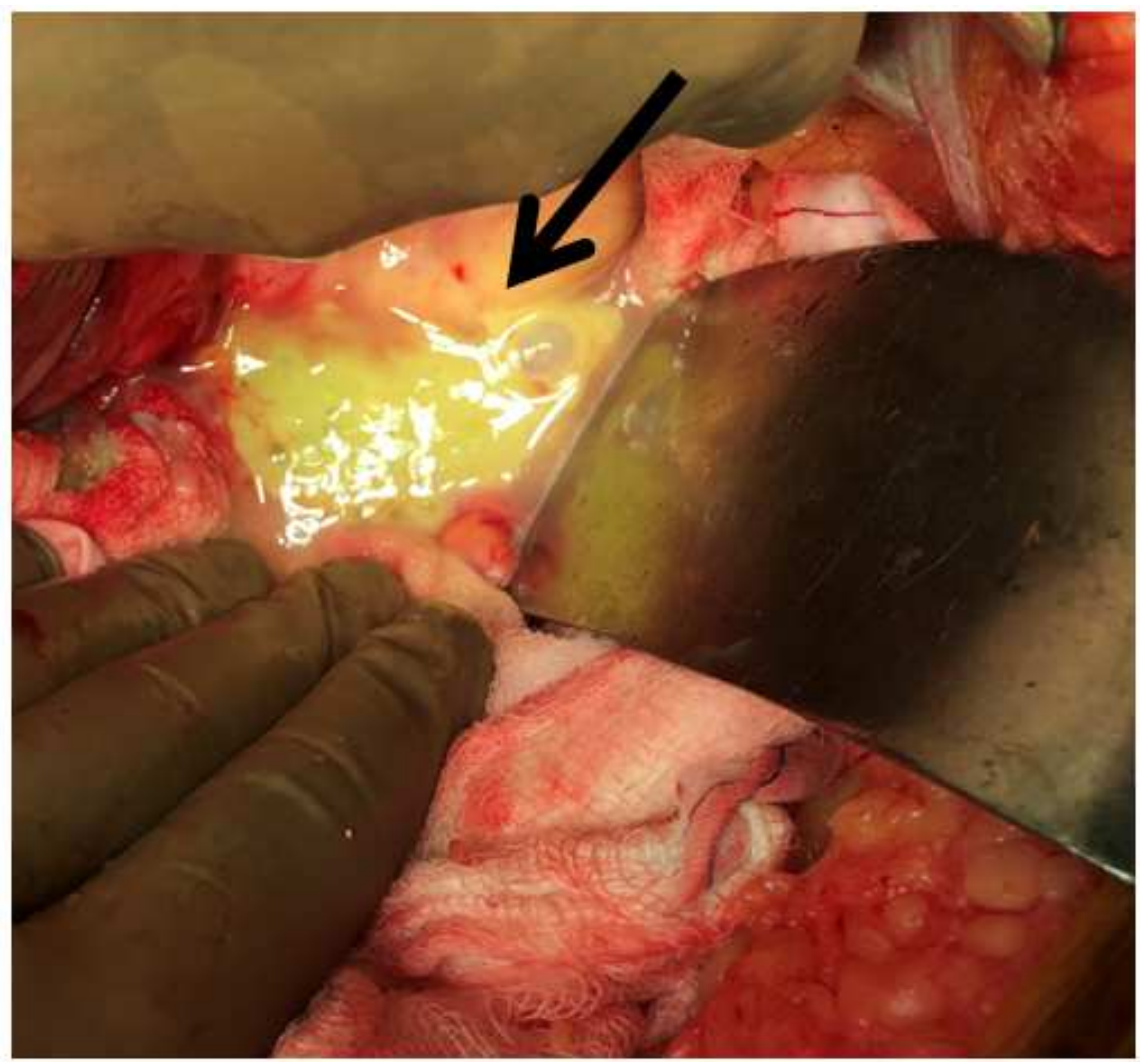

Fig. 4: Iatrogenic cyst rupture noted during the reflection of the posterior pelvic peritoneum, purulent material and daughter cyst were noted (arrow) shows daughter cyst

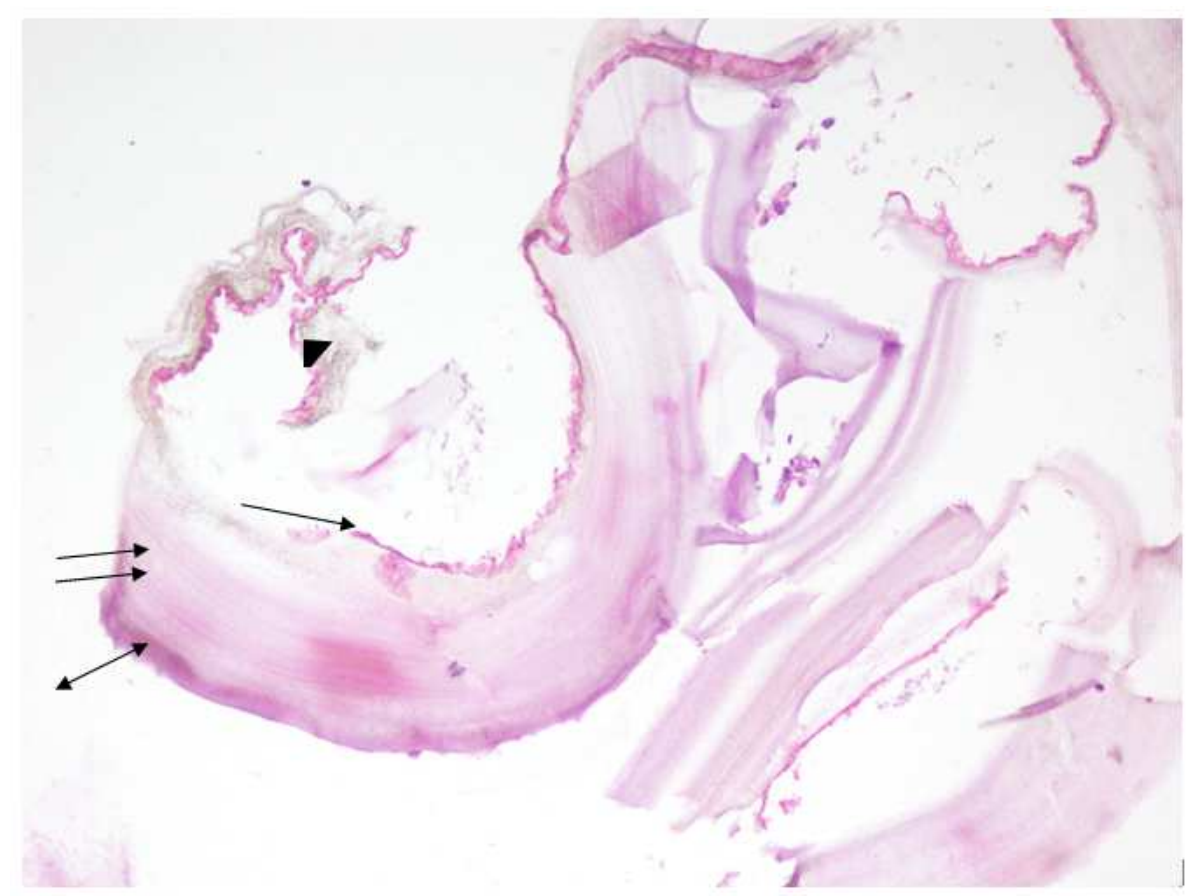

Fig. 5: Histopathology confirmed the diagnosis of hydatid cyst, showing the three layers and hydatid sand (arrow head), [germinal layer (arrow), laminar layer (two-arrow), thick adventitia (double arrow)] 


\section{Discussion}

Hydatid disease is an endemic disease, with canines the primary host and humans the intermediate host. The life cycle of Echinococcus granulosus may also involve sheep, cattle, goats and humans. This infection is transmitted orally via eggs shed in the faeces of infected animals. Liver, spleen and lungs are most common involved organs but it can involve any organ (Desai et al., 1999; Sherwani et al., 2015). Hydatid cyst consists of three layers: Pericyst, Ectocyst and Endocyst. Pericyst is the outer most covering and acts as a mechanical support. Bile ducts and blood vessels become incorporated within this structure. Ectocyst is the chitinous layer that acts as a barrier. Endocyst is the innermost layer and produces the infective clear hydatid fluid containing daughter cysts. (Pratap et al., 2018) HD is seen more frequently at the ages of 20 to 40 years and usually occurs in childhood and grows so slowly about $1-3 \mathrm{~cm}$ per year that the organism may take up to 20 years to reach considerable size (Ozkardes et al., 2014).

The retroperitoneal hydatid cyst is rare even in endemic areas (Aydinli et al., 2007). The overall frequency of peritoneal echinococcosis is approximately $13 \%$ of all cases (Mukerjee et al., 1973).

Retroperitoneal hydatid cyst are categorised into two types, primary and secondary (Desai et al., 1999; Sherwani et al., 2015). The retroperitoneal hydatid cyst is a rare entity even in endemic areas (Aydinli et al., 2007) first reported in 1958 by Lockhart and Sapinza (McPhail and Arora, 1967). Retroperitoneal hydatid cyst constituted $2.1 \%$ of all abdominal HC cases (Aydinli et al., 2007). Primary retroperitoneal hydatid cyst could be caused by haematogenous dissemination of protoscolices after bypassing the liver (veno-venous shunts within the liver and in the space of Retzius) and the lungs or by the gastrointestinal tract into the lymphatic system (McPhail and Arora, 1967; Barret, 1960). Dew and Waddle had favoured airborne transmission and direct implantation of the embryo in the bronchial mucosa as another possible mode of entry (Waddle, 1950; Spiliotis et al., 1999). This raises the possibility of an embryo of the parasite entering a venule after penetrating the bronchial mucosa and reaching the left side of the heart to involve other sites and thus bypassing the lung. But this remains largely theoretical and needs to be proved. Spontaneous, traumatic, or surgical rupture of a hepatic cyst may give rise to secondary retroperitoneal hydatid cyst (Spiliotis et al., 1999; Erçetin et al., 2008).

The differential diagnosis of retroperitoneal cysts includes soft tissue tumors, retroperitoneal abscess, cystic lymphangioma, embryonal cyst, ovarian neoplasms, teratoma and hydatid cyst (Erdem et al., 2009; Avc1 et al., 2013; Türkyilmaz et al., 2006) requires better evaluation before proceeding to definitive treatment.

The definitive diagnosis of a retroperitoneal hydatid cyst requires a combined assessment of clinical, radiological and serological analysis. Leukocystosis seen in infected hydatid cyst cases as in our case. Eosinophilia is seen in $25 \%$ cases. IgG ELISA has sensitivity of $95 \%$ and specificity of $94 \%$, IgG remain elevated for 4 years after therapy, hence IgM (ELISA) is better for post-treatment follow-up (Avc1 et al., 2013; Tepetes et al., 2007).

USG, CT \& MRI are standard investigations for diagnosis, percutaneous treatment and post-treatment follow-up. Ultrasonography is the first line imaging for the hepatic hydatid cyst and classify the stage of the hydatid cyst. The sensitivity of USG in diagnosing abdominal hydatid cyst ranges from $93 \%$ to $98 \%$ (Erdem et al., 2009). CT and MRI gives more precise information than USG regarding the morphology of the cyst, including size, location, number and relationships to surrounding structures, they display more precisely regarding the evidence of complications such as cysto-biliary communication, broncho-biliary communication. They are more superior to USG in detecting the extra hepatic hydatid cyst. The sensitivity of CT ranges from $90 \%$ to $97 \%$ (Tepetes et al., 2007). However, MRI, due to its multiplanar capabilities and the excellent contrast resolution for soft tissues, has a particular importance if the diagnosis of $\mathrm{HD}$ is questionable, because it is more accurate in demonstrating parietal features and defining anatomical relationships.

Although primary retroperitoneal hydatid cysts are rarely found, should have a high index of suspicion when findings suggestive of a cystic lesion in the retroperitoneum with abdominal pain as a symptom occurs in any patient especially in endemic areas of the disease (McPhail and Arora, 1967). Serological tests and better imaging should be considered in such cases prior to definitive treatment to prevent intra-operative complications. Mukerjee et al. (1973) reported nine cases, two had died due to anaphylactic reaction resulting from spillage during excision or biopsy done with the misdiagnosis of a retroperitoneal tumour.

The management of extrahepatic HD is based on considerations regarding the size, location and manifestations of the cysts and the overall health status of the patient. Asymptomatic small cysts can be treated with anti-helminthic drugs. In symptomatic and large hydatid retroperitoneal cysts, surgical resection is the only curative treatment. Surgical treatment can be either radical or conservative. Total cystectomy is the gold standard surgical modality (Avc1 et al., 2013; Tepetes et al., 2007; Hidatik, 2011).

The most important thing is to isolate the abdominal cavity with gauzes soaked in $20 \%$ hypertonic saline 
solution or $10 \%$ betadine for preventing the secondary hydatidosis and allergic reaction (Tepetes et al., 2007) laparoscopic approaches are also described. Spillage of the cyst contents must be avoided and scolicidal agents must be used in either conventional or laparoscopic technique. Although a variety of scolicidal agents have been used, there is no consensus on which is the best agent. Albendazole or praziquantel is indicated for inoperable and disseminated cases.

Percutaneous Aspiration, Injection and Reaspiration (PAIR) technique is another nonsurgical option (Tepetes et al., 2007). However, there have been some limitations for PAIR and it is only suitable for predominantly fluid and non-ruptured cysts (Ozkardes et al., 2014). PAIR could have been considered in our case if pre-operative diagnosis of hydatid cyst was made because the lesion was predominantly fluid filled and unruptured.

\section{Conclusion}

In conclusion, the differential diagnosis of the hydatid cyst should be considered for all the intraabdominal cystic lesions especially in endemic areas. Serological tests and better imaging should be considered in such cases prior to definitive treatment to prevent intra-operative complications. For confirmative diagnosis surgical removal of the cyst and histopathological examination of the resected specimen is necessary. Total cystectomy is the gold standard. When the complete resection is not feasible, de-roofing and drainage followed by adjuvant antihelminthic therapy must be performed to prevent secondary recurrence of the cyst.

\section{Acknowledgement}

The authors want to thank Prof.G Singh for helping with manuscript and Dr Santosh Irrinki for editorial assistance.

\section{Author's Contributions}

Hemanth Kumar: Performed the surgery and carried out the follow up study.

Anish Choudhary, Amaresh Aruni and N.V.K.N. Karthik: Drafted the manuscript and participated in the treatment of the patient. All authors read and approved the final mauscript.

\section{Ethics}

Informed verbal consent taken from patient for publishing the case report and accompanying images. Identity of the patient has not been disclosed.

\section{References}

Avc1, A., S. Bayil and R. Tekin, 2013. Left retroperitoneal hydatid cyst disease and the treatment approach. J. Microbiol. Infec. Dis. DOI: 10.5799/ahinjs.02.2013.04.0114

Aydinli, B., G. Ozturk, K.Y. Polat, S.S. Atamanalp and I. Ozbey et al., 2007. Extravisceral primary hydatid cyst of the retroperitoneum. ANZ J. Surgery, 77: 455-9. DOI: 10. /j.1445-2197.2007.04094.x

Barret, N., 1960. The anatomy and the pathology of multiple hydatid cysts in the thorax. Arris and Gale lecture. Ann Roy Col Surg Engl., 26: 362-79.

Desai, H.J., C.J. Bhatt and B.A. Dave, 1999. Imagesprimary spinal echinococcosis. Indian J. Radiol. Imaging, 9: 73.

Erçetin, C., M. Tükenmez, C. Dural, A. Poyanlı and A. Salmaslıoglu et al., 2008. Primary retroperitoneal hydatid disease mimicking retroperitoneal malignant tumor. Int. J. Infec. Dis., 12: 402-5. DOI: 10.1016/j.ijid.2007.11.004

Erdem, M.R., A. Akbaş, F. Onol, Y. Tanidir and S. Onol, 2009. An unusual retroperitoneal sero-negative hydatid cyst presenting with lower urinary tract symptoms. Türkiye. Parazitolojii. Dergisi, 33: 82-4.

Hidatik, T.E.R.K., 2011. Retroperitoneal Hydatid Cyst Simulating Irreducible Inguinal Hernia: Case Report. İrredükte Kasik Fitığını.

McPhail, J.L. and T.S. Arora, 1967. Intrathoracic hydatid disease. Diseases Chest, 52: 772-81.

DOI: $10.1378 /$ chest.52.6.772

Mukerjee, S., M. Nigam and J. Saraf, 1973. Primary retroperitoneal hydatid cyst. British J. Surgery, 60: 916-8.

Ozkardes, A.B., M. Tokac, F. Yetisir, B. Bozkurt and M. Kilic, 2014. Retroperitoneal hydatid cyst simulating irreducible inguinal hernia: case report. J. Clinical Analytical Med., 5: 243-5. DOI: 10.4328/JCAM.784

Ozturk, S., M. Unver, B. Kibar Ozturk, E. Kebapci and O. Bozbiyik et al., 2014. Isolated retroperitoneal hydatid cyst invading splenic hilum. Case Reports Surgery. DOI: 10.1155/2014/303401

Pratap, V., P. Ayyar and S. Prabhakar, 2018. Primary retroperitoneal hydatid cyst: A rare case with atypical presentation. Int. Surgery J., 5: 1959-61.

Sherwani, A.Y., A. Sarmast, A. Malik, M. Shah and Q. Fazl et al., 2015. Primary retroperitoneal hydatid cyst: a rare case report. Acta. Medica. Iranica, 53: 448-51.

Spiliotis, J.D., S.K. Kakkos, T. Petsas, D. Siablis and J.A. Androulakis, 1999. Inguinal swelling: A rare presentation of retroperitoneal hydatidosis. Eur. J. Surgery, 165: 75-6.

DOI: $10.1080 / 110241599750007559$ 
Tepetes, K., G. Christodoulidis, M. Spryridakis and K. Hatzitheofilou, 2007. Large solitary retroperitoneal echinococcal cyst: A rare case report. World J. Gastroenterology: WJG, 13: 6101. DOI: $10.3748 /$ wjg.v13.45.6101

Türkyilmaz, Z., K. Sönmez, R. Karabulut, I. Göl and A. Basaklar et al., 2006. Unusual localization of hydatid cyst. Acta Chirurgica Belgica, 106: 443-4.

Waddle, N., 1950. Pulmonary hydatid disease. A review of 478 cases reported in the louis barnett hydatid registry of the Royal Australasian College of Surgeons. Australian New Zealand J. Surgery, 19: 273-90. DOI: 10/j.1445-2197.1950.tb06569.x

\section{Abbreviations:}

hydatid disease HD

hydatid cyst HC

gastrointestinal GI

Jackson Pratt JP

Enzyme Linked Immuno-Sorbent Assay ELISA 